The abstracts are only available online, free of charge, under www.karger.com/doi/10.1159/000235631

\title{
2nd Congress of the International Society for Hemodialysis (ISHD)
}

August 28-30, 2009, Hong Kong

\section{Selected Abstracts}

Guest Editors

Sing-leung Lui, Hong Kong

Sydney Tang, Hong Kong

\section{Contents}

Hemodialysis Technology

Abstract 1

Abstracts 2-13

Complications of Dialysis

Abstracts 14-17

Home-Based Therapy

Abstracts 18-21

Continuous Therapy and ICU

Abstracts 22-26

Nursing Matters

Abstracts 27-29

Miscellaneous

Abstract 30

Author Index 


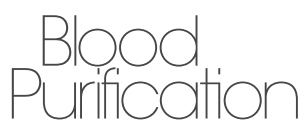

\section{Hemodialysis Technology}

1

\section{Prolonged Temporary Hemodialysis Catheter Survival with Copolymer Coating in Patients with End-Stage Renal Disease - PROCOP Study \\ Pascal Meier}

Centre Hospitalier du Centre du Valais, Sion, Switzerland

Recently, a novel coating technology that provides the surface with a micropatterned structure in a polymer system was proposed. We hypothesized that the improvement of blood-surface interaction by a reactive polymer film coating used in vitro might reduce thrombogenic events in the vascular access device and subsequently lead to prolonged catheter survival in the clinical setting. We therefore compared, in a randomized cross-over single-blinded study, the clinical application of two untunnelled temporary catheters (UTCs): one standard double lumen catheter (sDLC - GDK-1320) and one surface modified catheter (smDLC - SM-GDK-1320) with identical geometry and flow design with respect to catheter survival as the primary endpoint. All UTCs were inserted into the right internal jugular vein. An overall of 70 stable chronic HD patients were included to obtain sufficient statistical power. No anticoagulation outside the dialysis session was allowed, and each patient was on platelet anti-aggregation using aspirin $100 \mathrm{mg} / \mathrm{d}$. Efficacy endpoints were defined as the ability to complete HD and ability to achieve blood flow rates of ${ }^{3} 250$ $\mathrm{mL} / \mathrm{min}$. Safety endpoints were defined as the occurrence of allergic reactions, infection or bleeding. The clinical investigation revealed that both number of days before UTCs removal according to clinical requirements and number of treatments per catheter were significantly higher with smDLC as compared with sDLC ([mean $\pm \mathrm{SD}] 38 \pm 18$ vs. $20 \pm 9$ days; 27 vs. 16 treatments; $p=0.01$ ). The mean \pm SD blood flow rate was $295 \pm 29 \mathrm{~mL} / \mathrm{min}$ vs. $254 \pm 36 \mathrm{~mL} / \mathrm{min}$ for smDLC and sDLC, respectively (Qb: $\mathrm{p}=0.01)$. UTC malfunction occurred in $12 \%$ and $28 \%$ for smDLC and sDLC, respectively $(\mathrm{p}=0.001)$. Thrombosis of smDLC and sDLC was observed in 7.5 (2.3 episodes per 1,000 UTC days) vs. $21.3 \%$ (9.9 episodes per 1,000 UTC days, $\mathrm{p}=0.001)$. Micropatterned surface coating with a polyurethane polymer significantly increased UTC survival and the number of treatments per catheter.

\section{Outcomes and Clinical Care}

2

\section{Baseline Peritoneal Ultrafiltration Capability Predicts Survival of Hemodialysis Patients}

\author{
Cheuk Chun Szeto ${ }^{1}$, Bonnie Kwan ${ }^{1}$, Kai-Ming Chow ${ }^{2}$ \\ Chi-Bon Leung ${ }^{2}$, Philip Li,2 \\ ${ }^{1}$ The Chinese University of Hong Kong, Hong Kong, \\ 2Prince of Wales Hospital, Hong Kong
}

Background: Peritoneal transport status is a consistent predictor of mortality and morbidity in peritoneal dialysis (PD) patients. Since peritoneal transport status may actually reflect systemic vascular abnormality, we hypothesize that baseline peritoneal transport status may also predict the outcome of patients who have peritoneal failure and require conversion to hemodialysis.

Methods: We reviewed 72 consecutive patients (37 male) who failed PD patients and was converted to long-term hemodialysis in our unit between 1995 and 2007. Their baseline peritoneal transport status at the initiation of PD and survival during hemodialysis were retrieved and analyzed.

Results: The average age was $54.8 \pm 11.5$ years; duration of PD before conversion $29.9 \pm 24.9$ months. The average ultrafiltration volume (UF) was $0.35 \pm 0.18 \mathrm{~L}$; dialysate-to-plasma creatinine ratio at 4-hour was $0.61 \pm 0.14$; mass transfer area coefficient of creatinine was $8.73 \pm 4.13 \mathrm{ml} / \mathrm{min} / 1.73 \mathrm{~m}^{2}$. After conversion to hemodialysis, the two-year actuarial survival was $78.0 \%$ and $54.3 \%$ for patients with low and high peritoneal UF, respectively $(p=0.07)$. Further analysis showed that the time on PD before conversion to hemodialysis had significant interaction with peritoneal UF. For those who are converted to hemodialysis after at least 2 years of PD, the twoyear survival was $76.6 \%$ and $33.3 \%$ for low and high peritoneal UF, respectively $(\mathrm{p}=0.04)$, while for those who were converted to hemodialysis within 22 years of $\mathrm{PD}$, the two-year survival was $78.5 \%$ and $81.3 \%$, respectively $(\mathrm{p}=0.7)$.

Conclusions: A high baseline peritoneal UF status was associated with worse patient survival after conversion to hemodialysis, especially amongst patients who had been treated with PD for over 2 years. Our findings support the hypothesis that peritoneal transport status may reflect a systemic vascular dysfunction.

\section{KARGER \\ Fax +4161306 1234 \\ E-Mail karger@karger.ch}

www.karger.com
(C) 2009 S. Karger AG, Basel

0253-5068/09/0283-0276\$26.00/0

Accessible online at: www.karger.com/bpu 


\section{3}

\section{Brain Natriuretic Peptide Levels have Diagnostic and Prognostic Utility for Cardiac Events in Patients with Chronic Kidney Disease Admitted to the Intensive Care Unit}

\author{
Sung Gyun Kim¹, Son Jung Kim', Yong Rim Song ${ }^{1}$, \\ Hyung Jik Kim', Chun Soo Lim², Yon Su Kim², \\ Jung Sik Park ${ }^{3}$, Youngshin Shin ${ }^{4}$ \\ ${ }^{1}$ Hallym University Sacred Heart Hospital, Anyang, \\ Republic of Korea, ${ }^{2}$ Seoul National University College of \\ Medicine, Seoul, Republic of Korea, ${ }^{3}$ Asan Medical Center, \\ University of Ulsan, Seoul, Republic of Korea, \\ ${ }^{4}$ St. Vincent Hospital, The Catholic University of Korea, \\ Suwon, Republic of Korea
}

Objectives: To evaluate the diagnostic and prognostic utility of brain natriuretic peptide (BNP) in patients with chronic kidney disease (CKD) in the intensive care unit (ICU) setting.

Methods: All patients with CKD having serum creatinine $(\mathrm{Cr})$ $\geq 2.0 \mathrm{mg} / \mathrm{dl}$ admitted to the ICU were enrolled in this study. The CKD group was divided according to the presence or absence of acute decompensated heart failure (ADHF) into CKD + ADHF and CKD ADHF groups, respectively. Other patients with ADHF having low $\mathrm{Cr}$ $(<1.2 \mathrm{mg} / \mathrm{dl})$ in the coronary care unit were also recruited as a control group during the same period. BNP levels at the time of admission (admission BNP) were compared amongst these groups.

Results: Of 136 patients with CKD for whom data were available, including 58 on dialysis (42.6\%), 81 (59.6\%) had ADHF. Admission BNP were $2708.6 \pm 1246.9,567.9 \pm 491.7$ and $1418.9 \pm 1126.5 \mathrm{pg} /$ $\mathrm{ml}$ in the CKD + ADHF, CKD - ADHF and control groups $(\mathrm{n}=33)$, respectively $(\mathrm{P}=0.000)$. The optimal cutoff level was $1020.5 \mathrm{pg} /$ $\mathrm{ml}$ [area under the curve $(\mathrm{AUC})=0.944$ ] to detect ADHF from the receiver operating characteristic curve. This level was not associated with in-hospital mortality, all-cause death or a composite event (allcause death and/or new cardiac event). However, a borderline significant association was observed with new cardiac events [hazard ratio $(\mathrm{HR})=4.551 ; \mathrm{P}=0.078]$ during the follow-up period $(521.1 \pm 44.7$ days). Furthermore, continuous variables of BNP and BNP quartiles were significantly associated with new cardiac events in the multivariate Cox model $(\mathrm{HR}=1.001, \mathrm{P}=0.041 ; \mathrm{HR}=2.212, \mathrm{P}=0.018)$.

Conclusions: The findings suggest that the level of BNP at the time of admission may be a useful marker for detecting ADHF and predicting cardiac events in patients with CKD in the ICU setting.
4

\section{Substantial Improvement in Peritoneal Dialysis Survival Compared with Haemodialysis in the United States; A Longitudinal Trend Analysis: 1995-2004}

\author{
AG Stack 1 , A Nealon'1 H Nguyen ${ }^{1}$, CAM Wall ${ }^{1}$ \\ ${ }^{1}$ Regional Kidney Centre, Letterkenny, Donegal, Ireland, \\ ${ }^{2}$ Department of Renal Medicine, Adelaide and Meath \\ Hospitals, Dublin, Ireland
}

Introduction: Mortality differences exist between peritoneal dialysis (PD) and haemodialysis (HD). The aim of this study was to evaluate mortality differences and period trends between HD and PD in three successive calendar periods: 1995-1998, 1999-2001, and 2002-2004

Methods: National incidence data was collected on all new dialysis patients $(\mathrm{N}=1,003,305)$ who started treatment from 1995 to 2004 and were followed up to 4/10/2006. Interval Cox regression models allowing for non-proportional hazards evaluated the relative hazard of death by dialysis modality using intent-to-treat and as-treated analyses with a propensity based multivariable risk adjustment.

Results: During 2002-2004, adjusted mortality risks were significantly lower for PD vs HD for patients $<50$ yrs, and 50-70 yrs $(\mathrm{RR}=0.71$ and $\mathrm{RR}=0.82$ respectively $)$ and equivalent for patients $>70 \mathrm{yrs}(\mathrm{RR}=0.99)$. Across calendar periods, adjusted mortality risks were substantially lower for PD vs HD in 2002-2004 compared with 1999-2001 and 1995-1998 cohorts. In trend analysis, for Peritoneal Dialysis, adjusted mortality risks were significantly lower in more recent cohorts who started therapy $(1999-2001-\mathrm{RR}=0.90,95 \% \mathrm{CI}$ 0.86-0.94 and 2002-2004- RR $=0.79,95 \%$ CI 0.76-0.84) compared with earlier cohorts (1995-1998 $(\mathrm{RR}=1.00$, referent group).

For Haemodialysis, the improvement was less dramatic (1999$2001 ; \mathrm{RR}=0.98$, CI 0.97-0.98) and 2002-2004; RR $=0.95$, CI 0.94$0.96)$.

Conclusions: PD survival compared with HD has improved significantly and substantially from 1995-2006.

5

An Investigation Into the Quality of Life of Chronic Hemodialysis Patients Managed with Outcome Driven Protocols in South Africa

\author{
CL Becker $^{1}$,3 / Katz ${ }^{2}$, D Du Toit ${ }^{3}$ \\ ${ }^{1}$ National Renal Care, Johannesburg, South Africa, \\ ${ }^{2}$ Division of Nephrology, University of Witwatersrand, \\ Johannesburg, South Africa, ${ }^{3}$ Tshwane University of \\ Technology, Pretoria, South Africa
}

Objectives: End stage renal disease (ESRD) is a major health problem in South Africa associated with increased morbidity, mortality and decreased quality of life (QOL). The nephrology community is facing numerous challenges because of increasing numbers of patients on dialysis, limited resources and the increase in complexity 
of achieving better patient outcomes. This study aims to investigate patient's QOL on chronic hemodialysis in relation to clinical outcomes and patients knowledge of ESRD.

Methods: A descriptive study of ESRD patients undergoing hemodialysis, evaluating factors such as demographics, duration on dialysis, medical history, clinical indicators (hemoglobin, albumin, calcium, phosphate, dialysis dose) and the patient's understanding thereof. QOL was measured with the medical outcomes study 36 (SF36), which included a physical health composite score (PCS) and a mental health composite score (MCS). These factors, clinical scores and QOL measures were compared.

Results: One hundred patients from 10 units were evaluated, mean age $49 \pm 15$ years and on dialysis $2.6 \pm 1.8$ years. The PCS was $41.4+10.35$ and MCS $45+9.98$. A positive correlation was found between hemoglobin and PCS $(p=0.0093), \operatorname{MCS}(p=0.014)$ and the patients anemia knowledge $(\mathrm{p}<0.05)$. PCS was shown to be affected by their understanding of access care $(\mathrm{p}=0.0160)$, and hemodialysis complications $(p=0.0051)$. MCS was also found to be affected by the patients knowledge of their fluid management $(\mathrm{p}=0.0187)$ and influenced by family support $(\mathrm{p}=0.0092)$. Albumin $(38+5.2 \mathrm{~g} / \mathrm{L})$ and $\mathrm{Kt} / \mathrm{v}(1.1+0.3)$ did not affect QOL. Overall patients meeting the K/ DOQI guidelines in clinical indicators had an improved QOL (NS).

Conclusion: The study indicated that clinical indicators, patient's knowledge of ESRD and family support all impacted on a patient's QOL and their clinical outcome markers. Factors influencing QOL are complex, achieving guideline targets and educating patients have clear benefits for patients. Recognizing this will help clinicians manage patients better and improve outcomes on dialysis.

\section{6}

\section{Comparison of Two Years Outcome in Maintenance Dialysis Lupus Nephritis Patients}

Mingli Zhu, Yucheng Yan, Zhaohui Ni, Jiaqi Qian

Renal Division, Renji Hospital, School of Medicine, Shanghai Jiaotong University, Shanghai, China

Objective: To compare 2 years outcome of End-stage renal disease (ESRD) in lupus nephritis (LN) patients in different dialysis modality.

Methods: LN patients with maintenance dialysis in Renji Hospital were retrospective analysed from 1997 to 2006. All patients accepted dialysis for more than three months, and follow-up for two years more. Patients' clinical characteristics, mortality and morbidity were compared between hemodialysis (HD) and peritoneal dialysis (PD) patients.

Results: Total of $29 \mathrm{LN}$ patients were enrolled, one and two years survival of all patients were $100 \%$ and $86 \%$. There were 10 patients in HD group and 19 in PD group. At the baseline of dialysis, there was no difference between two groups, including sex (male $30 \%$ vs $31.6 \%, \mathrm{P}>0.05)$, age $(34.50 \pm 8.58$ vs $41.79 \pm 15.55$ years, $\mathrm{P}>0.05)$, hemoglobin $(\mathrm{Hb})(61.00 \pm 15.35$ vs $66.00 \pm 12.13 \mathrm{~g} / \mathrm{L}, \mathrm{P}>0.05)$ and serum albumin $(29.40 \pm 3.14$ vs $30.61 \pm 6.80 \mathrm{~g} / \mathrm{L}, \mathrm{P}>0.05)$, ESR $(72.33 \pm 41.33$ vs $86.30 \pm 40.51 \mathrm{~mm} / \mathrm{h}, \mathrm{P}>0.05), \mathrm{C}_{3}(0.52 \pm 0.14$ vs $0.72 \pm 0.27 \mathrm{mmol} / \mathrm{L}, \mathrm{P}>0.05)$. There were no difference in the incidence of SLE relapse $(20 \%$ vs $15.8 \%, \mathrm{P}>0.05)$. During the first two years of dialysis, patients in two groups had the similar incidence of infectious complication and CVD. There was no difference in $\mathrm{Hb}$ and immunosuppression dose between two groups. $\mathrm{Hb}$ and serum albumin level at 6, 12, 18 and 24 month were higher than baseline in both group (each $\mathrm{P}<0.05$ ). In the second year, the serum albumin was lower in PD group than in HD group $33.03 \pm 5.00$ vs $39.78 \pm 2.74$ $\mathrm{g} / \mathrm{L}, \mathrm{P}<0.05)$. Patient mortality two years after dialysis was similar in $\mathrm{PD}$ and HD group $(\mathrm{P}>0.05)$. However, in $\mathrm{PD}$ group, 4 patients switched to HD because of severe peritonitis, and $2(9.5 \%)$ received renal transplantation, while none of HD patients dropout.

Conclusion: Anemia and hypoalbuminemia can be improved after starting dialysis in LN patients. The incidence of infection, lupus relapse and CVD are similar in HD and PD patients.

\section{7}

\section{Prognostic Value of Access Blood Flow in Haemodialysis Patients}

XJ Che, Zh H Ni, JO Qian, YC Yan, LYGu

Renal Division, Renji Hospital, Shanghai, China

Objective: To investigate the relationship between access blood flow (Qa) and vascular access function by measurement of Qa in maintenance haemodialysis patients plus follow-up of their vascular access function.

Methods: Qa were measured using the Transonic HD02 Monitor. The patients who suffered from $\mathrm{Qa}<600$ or $>1,800 \mathrm{ml} / \mathrm{min}$ were measured the vascular access by colour Doppler ultrasonography in an attempt to rule out the possibility of vascular stenosis. Vascular access function was carefully monitored through follow-up for the exploration of its relationship with Qa.

Results: 125 patients succeeded in monitoring the vascular access. The mean Qa was $995.2 \pm 637.5 \mathrm{ml} / \mathrm{min}, \mathrm{Qa}<600 \mathrm{ml} /$ min was found in 32 patients $(25.6 \%), \mathrm{Qa}=600-1800 \mathrm{ml} / \mathrm{min}$ in 83 patients $(66.4 \%), \mathrm{Qa}>1800 \mathrm{ml} / \mathrm{min}$ in 10 patients $(8 \%) .10$ patients presented low Qa measured by color Doppler ultrasonography. In these patients, stenosis at the inosculated part of blood vessel was found in 4 patients $(40 \%)$, intima hyperplasia at the site frequently punctured in 2 patients $(20 \%)$, vein shunt in 2 patients $(20 \%)$, and the vascular access was normal in 2 patients $(20 \%)$. The radial arteries in all of the 7 patients whose Qa was too high were dilated. In 36 months' follow-up, vascular access occlusion was found in 13 patients $(40.6 \%)$ of the low Qa group who underwent re-operation, while 7 patients $(8.4 \%)$ of the normal Qa group and no patient $(0 \%)$ of the high Qa group. Correlation analysis showed Qa and vascular access prognosis were negatively correlated $(r=-0.318, P<0.001)$ and multiple regression analysis demonstrated the impact of Qa upon vascular access prognosis $(\beta=-0.316, \mathrm{P}=0.005)$.

Conclusions: Ultrasound dilution technology has its prognostic value in vascular access function by surveillance of Qa among haemodialysis patients. Lower Qa should give rise to greater possibility of vascular access occlusion which prove to be a good way for the surveillance of vascular access. 


\section{8}

\section{The Effects of Proactive Iron Protocol Implementation on Achieving Clinical Guideline Targets for Anaemia in a Satellite Haemodialysis Patient Cohort}

\section{KYong $^{1}$, L Kairaitis $^{1}{ }^{1}$, E Yuill $^{1}$}

${ }^{1}$ Department of Renal Medicine, Sydney West Area Health Service, Westmead Hospital, Hawkesbury Road, Westmead 2145, New South Wales, Australia, ${ }^{2}$ School Of Medicine, University of Western Sydney, New South Wales, Australia

Aim: Anaemia management with erythropoietic-stimulating agents (ESAs) and intravenous iron replacement in haemodialysis patients poses several cliical challenges including maintaining stable haemoglobin $(\mathrm{Hb})$ levels within target ranges while achieving lowest effective ESA dose. This manuscript describes the effect of implementing proactive protocol driven adjustments for iron and ESAs in a cohort of maintenance haemodialysis patients.

Methods: This was a retrospective cohort study of 48 satellite haemodialysis patients examined from 2004 to 2006 with protocol implementation in 2005. Baseline haemoglobin $(\mathrm{Hb})$, transferrin saturations (TSAT), ferritin values and ESA dosage were obtained during 2004 prior to protocol implementation (2005). Followup data was collected in 2006 and compared to baseline values in reference to specified target ranges in the 2004 Caring for Australasians with Renal Impairment (CARI) guidelines.
Results: Haemoglobin values were improved with $54 \%$ patients in 2006 achieving targets versus $43 \%$ patients in 2004 . Similar improvement was seen in iron indices with 79\% (2006) versus 67\% (2004) achieving TSAT targets and 90\% (2006) versus 75\% (2004) achieving ferritin targets. Odds ratios (OR) for values falling within the target range during the followup period compared to baseline period were 1.63 ( $\mathrm{Hb}$; p-value $0.037 ; 95 \%$ CI 1.03 to 2.57), 1.90 (TSAT; p-value $0.006 ; 95 \%$ CI 1.20 to 3.01 ) and 3.72 (ferritin; $p$-value 0.003 ; $95 \%$ CI 1.57 to 8.83 ) respectively. There was a non-significant trend toward lower average ESA use (p-value 0.07).

Conclusion: This study demonstrates a clinical framework in which proactive protocol-driven adjustment of ESA and iron dosages versus reactive response to pathology results can improve haemoglobin and iron management in haemodialysis patients. Benefits include increased concordance with historical anaemia targets and increased stability of haemogobin parameters. Optimising ESAs to achieve lowest effective dose can aid in limiting increasing costs of anaemia management and improve clinical outcomes in the haemodialysis population.

Table 1. Odds ratios (OR) are demonstrated above. The odds of a followup reading (2006) falling within recommended target ranges was significantly higher compared to initial readings (2004) for haemoglobin, TSAT and ferritin

\begin{tabular}{lllll}
\hline & Odds ratio & $\begin{array}{l}\text { Lower 95\% } \\
\text { limit }\end{array}$ & $\begin{array}{l}\text { Upper 95\% } \\
\text { limit }\end{array}$ & p-value \\
\hline Haemoglobin & 1.63 & 1.03 & 2.57 & 0.037 \\
TSAT & 1.96 & 1.20 & 3.20 & 0.007 \\
Ferritin & 5.08 & 1.93 & 13.41 & 0.001 \\
\hline
\end{tabular}

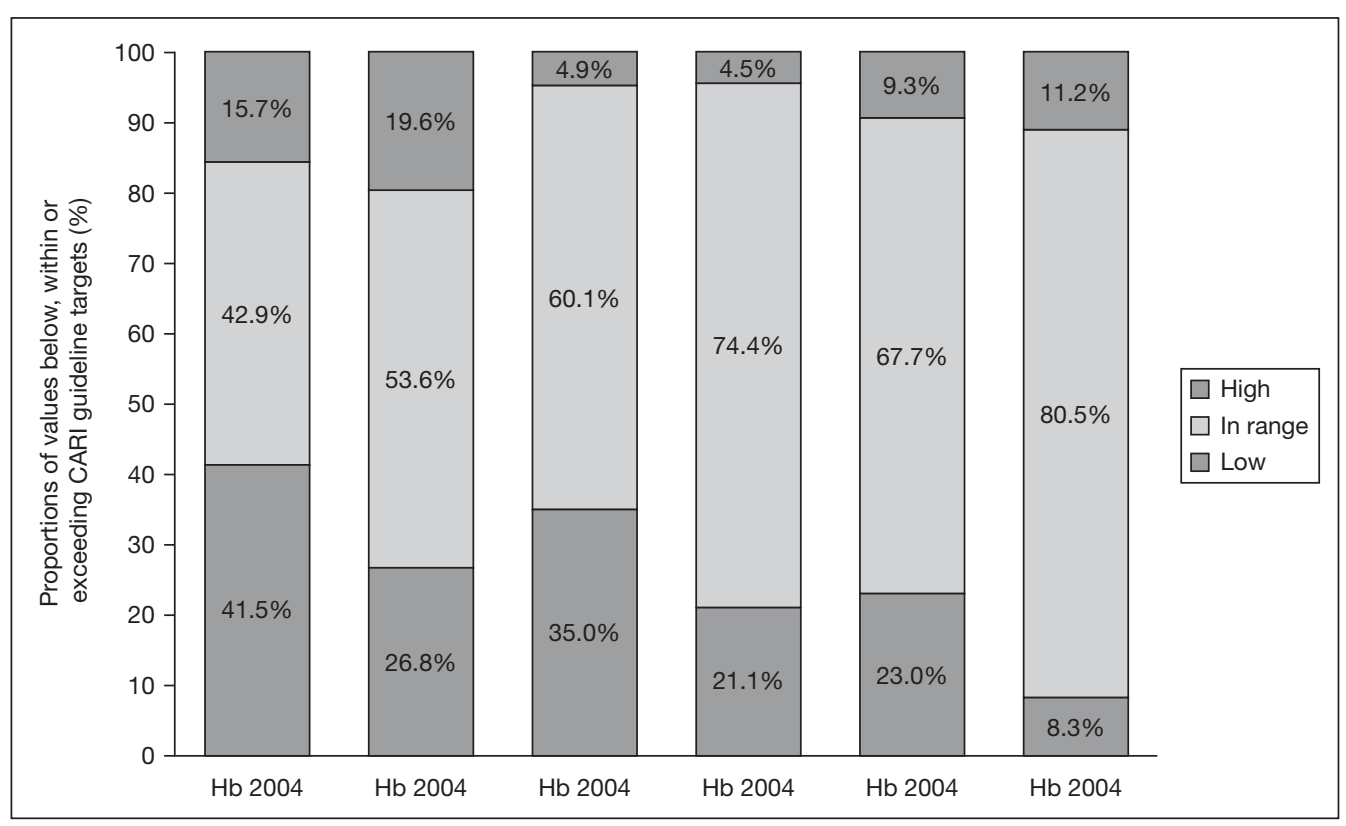

Figure 1. Graph demonstrating distribution of proportions of values which were below CARI targets (lower dark), achieved CARI targets (white), and exceeded CARI targets (upper dark). There was overall improvement in proportions of values successfully maintained within recommended CARI guideline targets over the time period 


\section{9 \\ Post-Parathyroidectomy Hypocalcemia in Hemodialysis Patients with Renal Hyperparathyroidism: Incidence and Risk Factors}

CHR Tan, OL Yong, HL Choong

Singapore General Hospital, Singapore, Singapore

Aims: Despite advances in medical management, parathyroidectomy (PTX) plays an important role in the treatment of refractory renal hyperparathyroidism. Post-PTX hypocalcemia is a common and serious complication. This study is to evaluate the incidence, severity and risk factors of hypocalcemia post-PTX.

Methods: Data was retrieved from a database consisting of all hemodialysis patients who had undergone PTX from 2007-08. Those risk factors investigated were pre- and post-PTX parathyroid (iPTH), calcium, phosphorus and serum alkaline phosphatase (ALP) levels.

Results: Fifty hemodialysis patients underwent total PTX with deltoid implantation and had received post-op intravenous calcium, oral calcium and vitamin D analogue. Post-PTX hypocalcemia was defined as a corrected serum calcium value $<2.10 \mathrm{mmol} / \mathrm{L}$ within first 3 post-operative days. Hypocalcemia developed in $24 \%(12 / 50)$ of post-PTX patients (serum calcium level of $1.98+0.12 \mathrm{mmol} / \mathrm{L}$ ) and was associated with an increased length of hospital stay $(10.2+3.8$ vs $7.0+1.9$ days) compared with patients without hypocalcemic episodes. Higher Pre-PTX iPTH and ALP levels were the risk factors identified (iPTH $290+63 \mathrm{pmol} / \mathrm{L}$ in hypocalcemic group vs $205+85 \mathrm{pmol} / \mathrm{L}$ in those without hypocalcemia; ALP $425+201 \mathrm{U} / \mathrm{L}$ in hypocalcemic group vs $290+143 \mathrm{U} / \mathrm{L}$ in those without hypocalcemia). Subgroup analysis showed that pre-PTX iPTH $>250 \mathrm{pmol} / \mathrm{L}$ and ALP $>400 \mathrm{U} / \mathrm{L}$ predict post-operative hypocalcemia with an OR 11.1 and 6.5 respectively.

Conclusion: Hypocalcemia post-PTX is a serious and common complication in hemodialysis patients and pre-operative markedly elevated iPTH and ALP levels predict post-operative hypocalemic events. It is recommended that more intensive monitoring and aggressive replacement of calcium in this group of patients postoperatively.

\section{0}

\section{Prevalence of Micronutrients Deficiency in Hospitalized Dialysis Patients}

\author{
Rama Tripathi ${ }^{1}$, Amit Srivastava ${ }^{2}$, Anurag Singh1, \\ R.K. Sharma', Anupma Kaul ${ }^{1}$ \\ ${ }^{1}$ Sanajay Gandhi Post Graduate Institute of Medical \\ Science, Lucknow, U.P., India, ${ }^{2}$ Central Drug Research \\ Institute, Lucknow, U.P., India
}

Micronutrients deficiency exists in most of the uremic patients, and with the degree of renal failure this deficiency increases. Dialysis further worsens this condition by losses of water soluble vitamins like vit $\mathrm{B} 12$, folate, and other nutrients like $\mathrm{Se}, \mathrm{Zn}$ and enzyme regulatory compounds. Purpose of this study is to focus on-(i) Prevalence of micronutrient deficiency in hospitalized patients. (ii) Relationship between poor nutritional status and low micronutrient intake in dialysis patients. Fourty six hospitalized dialysis patients (12 females, 34 males, mean age $47.3 \pm 14.7$ years) were included in this study cross section ally. SGA \& MIA were assessed by standard format. Biochemical parameters i.e. BUN, Cr., S.albumin, Ca, Po4 etc were recorded by hospitals different labs, Anthropometric parameters determined by Skin fold calipers. Dietary micro and major nutrients were evaluated by $24 \mathrm{hr}$ dietary recall method .MIA (malnutrition inflammation assessment) score format also contains serum parameters like serum albumin and TIBC. The mean weight was $57.5 \pm 11.5 \mathrm{~kg}$ and BMI was $21.3 \pm 3.6 \mathrm{~kg} / \mathrm{m} 2$ of all patients. SGA and MIA score mean value was $19.6 \pm 4.8 \& 16.3 \pm 4.4$, respectively. The observed mean value for different major and micronutrients were ; dietary Protein $(52 \pm 14.4$ gm, compare to recommended $70 \mathrm{gm})$, Iron $(6.8 \pm 2.6 \mathrm{mg}$, recom. 28 $\mathrm{mg})$, Folate $(193.2 \pm 66.9 \mu \mathrm{gm}$-recom.200 $\mu \mathrm{gm}), \mathrm{Zn}(3.6 \pm 1.3 \mathrm{mg}$ recommended $15 \mathrm{mg}), \mathrm{Se}(0.39 \pm 0.17 \mu \mathrm{gm}$ recommended $60 \mu \mathrm{gm}) \&$ $\mathrm{B} 12(0.60 \pm 0.21 \mu \mathrm{gm}$-recommended $1 \mu \mathrm{gm})$, which is much lower than the recommended intake for dialysis patients and low micronutrient intake has significant inverse correlation with uric acid ,high SGA \& MIA scores. Above study shows that those patients who has poor nutritional status and taking low micronutrient diet has high MIA and SGA score and also shows significant relationship between low intake of trace elements through diet and malnutrition of dialysis patients. Further basic and clinical research is required to establish whether how significantly high micronutrients through diet or supplements slow down the malnutrition status among dialysis patients.

11

Rationale and Design of the ACTIVE Dialysis Trial: A Multicentre, Unblinded, Randomised Controlled Trial of Extended vs Standard Duration of Dialysis in Patients with End Stage Kidney Disease
A Cass $^{1}$, MJ Jardine 1 , MP Gallagher ${ }^{1}$, KHoward $^{2}$ CHawley $^{3}$, P Kerr' , J Agar ${ }^{5}$, J Eris ${ }^{6}$, V Perkovic ${ }^{1}$
${ }^{1}$ The George Institute for International Health, Sydney, NSW, Australia, ${ }^{2}$ University of Sydney, Sydney, NSW, Australia, ${ }^{3}$ Princess Alexandria Hospital, Brisbane, Qld, Australia, ${ }^{4}$ Monash Medical Centre, Melbourne, Vic, Australia, ${ }^{5}$ The Geelong Hospital, Geelong, Vic, Australia, ${ }^{6}$ Statewide Renal Services, Sydney, NSW, Australia

Introduction and Aims: Registry data suggests that increased weekly dialysis hours are associated with lower mortality for people with end stage kidney disease (ESKD). This association is thought to be only partially explained by measured confounders. There is a paucity of randomized controlled trial (RCT) data exploring the benefits and harms of extended dialysis hours. The ACTIVE Dialysis trial will compare the effects on quality of life, clinical outcomes and cost-utility of two HD regimens: extended HD treatment vs. standard HD treatment.

Methods: The study is a multicentre unblinded RCT with an intervention period of 12 months. It will include 200 incident or prevalent adults with ESKD receiving HD treatment either 3 times 
weekly or alternate daily with no definite expectation of ceasing HD within 12 months. Eligible individuals will be randomised to treatment for 12 months with either extended hours HD (expected average 24-28 hours over 3-3.5 sessions per week) or standard hours of HD (expected average 12-15 hours). The exact dialysis prescription and the use of background therapies will be determined by the treating physician. The primary endpoint is the change in quality of life at 12 months as measured by the EuroQOL quality of life instrument. A formal analysis will be performed to estimate the relative cost effectiveness of the two interventions from the perspective of the health care provider in terms of cost per quality adjusted life year. Other secondary endpoints include a composite cardiovascular endpoint, patient safety, technique survival, cardiac geometry, difference in hospitalisation rates, blood pressure, access related events and survival.

Conclusions: ACTIVE Dialysis will provide important clinical outcome and economic data regarding the effects of a practical and widely applicable form of extended dialysis.

\section{2 \\ Blood Pressure Evolution during the First 6 Months of Hemodialysis Therapy. Lessons from the Dry Weight Method}

\author{
C Chazot, C Vo-Van, JC Terrat, TVanel, C Lorriaux, \\ JM Hurot, B Mayor, G Jean \\ Centre de Rein Artificiel, Tassin, France
}

Hypertension associated with extracellular fluid (ECF) overload contributes to the high rate of cardiovascular mortality in the dialysis setting.

Between 2000 and 2008, 196 ESRD patients (63.2 \pm 15.8 y.o.; $\mathrm{F} / \mathrm{M}=70 / 126 ; 28.1 \%$ with diabetes), started HD therapy in our unit and survived at least 6 months. They were prescribed mainly with low flux polysulfone dialyzer and $6.7 \pm 1.4$ hours per session, 3 times/ week. Dry weight method was applied. Table 1 reports the body weight (BW) evolution during the probing phase (W1 to W9, the usual period for probing) and BP decrease between the HD start and 6 months (W1 to W27), the usual time to reach a BP plateau after HD start.

The average postdialysis BW decreases between $\mathrm{W} 1$ and $\mathrm{W} 9$ was $-2.8 \pm 6.4 \%$. The postdialysis BW variation in $\%$ at W9 significantly correlated with the absolute variation of systolic BP (SBP) and pulse pressure (PP) at W27. Patients in the lower tertile of SBP at W1 had a stronger trend for lower survival than in the 2 other tertiles $(p=0.053)$. Patients in the higher tertile of diastolic BP and mean arterial pressure at W1 had a better survival than in the two lower ones (respectively $\mathrm{p}=0.01$ and 0.023 ). However, there was no difference in survival according to tertiles of SP, DBP, MBP and PP at W27

These data confirm the efficiency of ECF removal on correction of hypertension. After 6 months of HD therapy and ECF overload correction, the relationship between BP levels and survival is overruled. Relationships between ECF overload, cardiac function and BP levels may explain these findings.

13

\section{Increased Oxidative Stress and Endothelial Dysfunction in Hepatitis C Virus Infected Chronic Hemodialysis Patients}

\author{
N Reddy Pamidi, K Dakshina Murty, V Ram Rapur, \\ $P$ Reddy Chinthaparthi \\ Nizams Institute of Medical Sciences, Hyderabad, Andhra \\ Pradesh, India
}

Background: Increased cardiovascular disease (CVD) in hemodialysis leads to increased morbidity and mortality. In addition to traditional risk factors for CVD, non-traditional risk factors like calcium phosphate product, micro inflammatory state and oxidant stress contribute. Increased incidence of Hepatitis $\mathrm{C}$ virus (HCV) infection has added to this oxidative burden. Among methods for assessment of arterial damage, measurements of carotid-femoral pulse wave velocity (PWV), Aortic augmentation (AIx) and oxidative stress are considered to predict cardiovascular risk. This study is done to assess effect of HCV infection on oxidative stress markers and its correlation to endothelial function in HD patients.

Methods: A prospective cross sectional study, done with IEC approval and informed consent. Patients were divided into HCV negative $(n=15)$, HCV positive $(n=24)$ and compared with healthy age matched control group $(n=15)$. Serum levels of malondialdehyde as thiobarbituric acid reactive substance (TBARS), protein carbonyl content are measured as marker of oxidant stress, protein sulfohydryl groups as antioxidant marker and were correlated with nitric oxide levels (NO), Carotid-Femoral pulse wave velocity and Aortic augmentation done by SphygmoCor, AtCor Medicals, Australia

Results: Baseline parameters are comparable among all groups $(p>0.05)$. As the duration of HD is significantly higher in HCV positive $(\mathrm{p}<0.001)$ they were divided into 2 sub-groups to nullify the effect of HD duration.

Conclusion: HD treatment is associated with raise in serum TBARS level and serum protein carbonyl content and a decrease in serum protein sulfhydryl content and NO levels. HCV infection augments the adverse effects of HD on serum markers of oxidative stress

Table 1 (for Abstract 12). BP and BW evolution during the first 6 months of HD therapy

\begin{tabular}{|c|c|c|c|c|c|c|c|c|}
\hline & Systolic BP & Diastolic BP & Mean & Arterial & Pressure & Pulse & pressure & Postdialysis \\
\hline $\begin{array}{l}\text { BW W1 } \\
\text { W9 }\end{array}$ & $143.2 \pm 24.8$ & $76.2 \pm 14.9$ & $98.5 \pm 16.8$ & $67.1 \pm 17.8$ & $\begin{array}{l}69.8 \pm 14.6 \\
67.7 \pm 14.0 \dagger\end{array}$ & & & \\
\hline W27 & $132.9 \pm 20.3 *$ & $70.7 \pm 3.5^{*}$ & $91.4 \pm 14.4 *$ & $62.3 \pm 15.1 *$ & $68.6 \pm 14.9 *$ & & & \\
\hline
\end{tabular}

*: $\mathrm{p} \leq 0.0001$ between $\mathrm{W} 1 \& \mathrm{~W} 27 ; \dagger: \mathrm{p}<0.0001$ between $\mathrm{W} 1 \& \mathrm{~W} 9$ 
Table for Abstract 13

\begin{tabular}{lllll}
\hline Parameter & Control & Hev Negative & Hev Positive $\leq 2$ Yrs & Hev Positive $\geq 2$ Yrs \\
\hline Serum TBARS $(\mathrm{nmol} / \mathrm{L})$ & $3.54 \pm 0.32$ & $8.82 \pm 2.21$ & $12.59 \pm 3.68^{*}$ & $11.01 \pm 2.75^{*}$ \\
Protein carbonyl $(\mathrm{nmol} / \mathrm{L})$ & $0.66 \pm 0.21$ & $1.23 \pm 0.38$ & $1.77 \pm 0.89^{*}$ & $2.00 \pm 0.76^{*}$ \\
Protein sulfhydryl $(\mu \mathrm{mol} / \mathrm{mL})$ & $2.00 \pm 0.76$ & $302.3 \pm 99.54$ & $234.8 \pm 77.9^{*}$ & $233.1 \pm 77.8^{*}$ \\
Serum NO $(\mu \mathrm{mol} / \mathrm{L})$ & $33.67 \pm 5.78$ & $8.18 \pm 4.12$ & $9.13 \pm 3.51$ & $11.91 \pm 0.76^{*}$ \\
Cf PWV $(\mathrm{m} / \mathrm{s})$ & $7.4 \pm 1.19$ & $11.0 \pm 1.45$ & $31.0 \pm 6.41$ & $3.0 \pm 1.35^{*}$ \\
Alx $(\%)$ & $8.7 \pm 0.63$ & $28.7 \pm 4.64$ & & $37.5 \pm 5.27^{*}$ \\
\hline
\end{tabular}

${ }^{*} \mathrm{p}<0.001$ with HCV negative.

and increases the endothelial dysfunction. However HCV infection seems not to cause any additional decrease in serum NO levels.

\section{Complications of Dialysis}

\section{4 \\ The Effect of Treg/Th17 Functional Imbalance on Development and Outcome of Adverse Cardiovascular Events in Uremia Patients with Maintenance Hemodialysis}

\author{
Jian Bin Zhang, Hua Gan, Xiao Gang Du \\ ChongQing Medical University, ChongQing, China
}

Objective: Adverse cardiovascular events resulting from accelerated atherosclerosis are the leading cause of mortality in uremic patients on maintenance hemodialysis (MHD). The aim of the present study was to assess the Treg/Th17 pattern in uremic patients on MHD and to explore the significance of the imbalance of Treg/Th17 function in the development and outcome of acute cardiovascular events.

Methods: Forty-two MHD patients were evaluated: 22 MHD patients with a history of acute cardiovascular events served as MHD1 group and 20 MHD patients without acute cardiovascular events served as MHD2 group. Thirty patients with advanced chronic kidney disease (CKD) without acute cardiovascular events just before hemodialysis therapy served as CKD control patients and thirty healthy volunteers as normal controls. The frequency of Treg and Th17 cells were measured by flow cytometry, ROR $\gamma$ t and Foxp3 expression were measured by real-time RT-PCR, serum cytokines and C-reactive protein (CRP) were detected by ELISA and immunoturbidimetry.

Results: Patients with CKD revealed obvious imbalance of Treg/Th17 function, displaying increased peripheral Th17 numbers, Th17-related cytokines(IL-17,IL-6 and TNF-a) and transcription factor (ROR $\gamma \mathrm{t}$ ) mRNA levels $(\mathrm{p}<0.05$ between healthy subjects and patients; $p<0.05$ between MHD1 and MHD2,respectively), while significantly decreased Treg numbers, Treg-related cytokines (IL-10,TGF- $\beta 1$ ), and Foxp3 mRNA levels ( $\mathrm{p}<0.05$ between healthy subjects and patients; $p<0.05$ between MHD1 and MHD2). The imbalance was more pronounced in MHD2 group while there was no significant difference between the MHD1 and CKD control patient groups. We also observed that the imbalance of Treg/Th17 was not only consistent with bad heart function but also correlated with a microinflammatory state.

Conclusion: In CKD patients an imbalance of Th17/Treg cells exists due to uremia. The imbalance of Th17/Treg may act synergistically with microinflammation on immune-mediated atherosclerosis and contribute to the high incidence of acute cardiovascular events.

\section{5 \\ Outcome of the Haemodialysis Catheter Related Bacteraemia Treated with Systemic Antibiotics and Antibiotic Lock Therapy - A Single Centre Experience

\author{
SK Chan, CK Chan, CC Chow, HKS Lo, KLS Mo, CK Wong, \\ KS Wong \\ Pamela Youde Nethersole Eastern Hospital, Hong Kong
}

Background: Tunneled cuffed dialysis catheters (CTC) are increasingly used for haemodialysis worldwide. One of the major complications related to the use of CTC is catheter related bacteraemia (CRB). Biofilm formation is believed to be the reason behind difficult eradication of $\mathrm{CRB}$ with antibiotics alone and therefore, the treatment of CRB should include both antibiotics and biofilm eradication. Antibiotic lock therapy (ALT) was thus devised to serve this purpose.

Methods: The outcome of ALT in addition to the standard systemic antibiotics in treating CRB was retrospectively reviewed in a cohort of haemodialysis patients using CTC in a single centre. Both efficacy and safety of ALT were studied and comparison was made with historical control. Treatment success was defined as fever subsided within three days of starting appropriate systemic antibiotics and ALT together with no relapse of CRB caused by same organism within 30 days of completion of treatment. Those presented with severe sepsis, cases of relapse, concurrent tunnel infection and no appropriate antibiotic for locking were excluded from analysis.

Results: ALT was successful in 30 out of 32 episodes in the study group and 4 out of 8 episodes in the control group $(p<0.01)$. Length of hospitalization was also significantly reduced. There was no major complication with ALT and the prevalence of CRB per 1000 catheter days was not increased after implementation of ALT. Subgroup analysis on CRB caused by Staphyloccocus aureus also showed better outcome in study group $(\mathrm{p}<0.05)$.

Conclusions: Co-therapy of ALT and systemic antibiotics may have advantage over systemic antibiotics alone, although randomized controlled trial is required to make a definite proof. Selection of appropriate patients is the key to high success rate and ensuring patient 


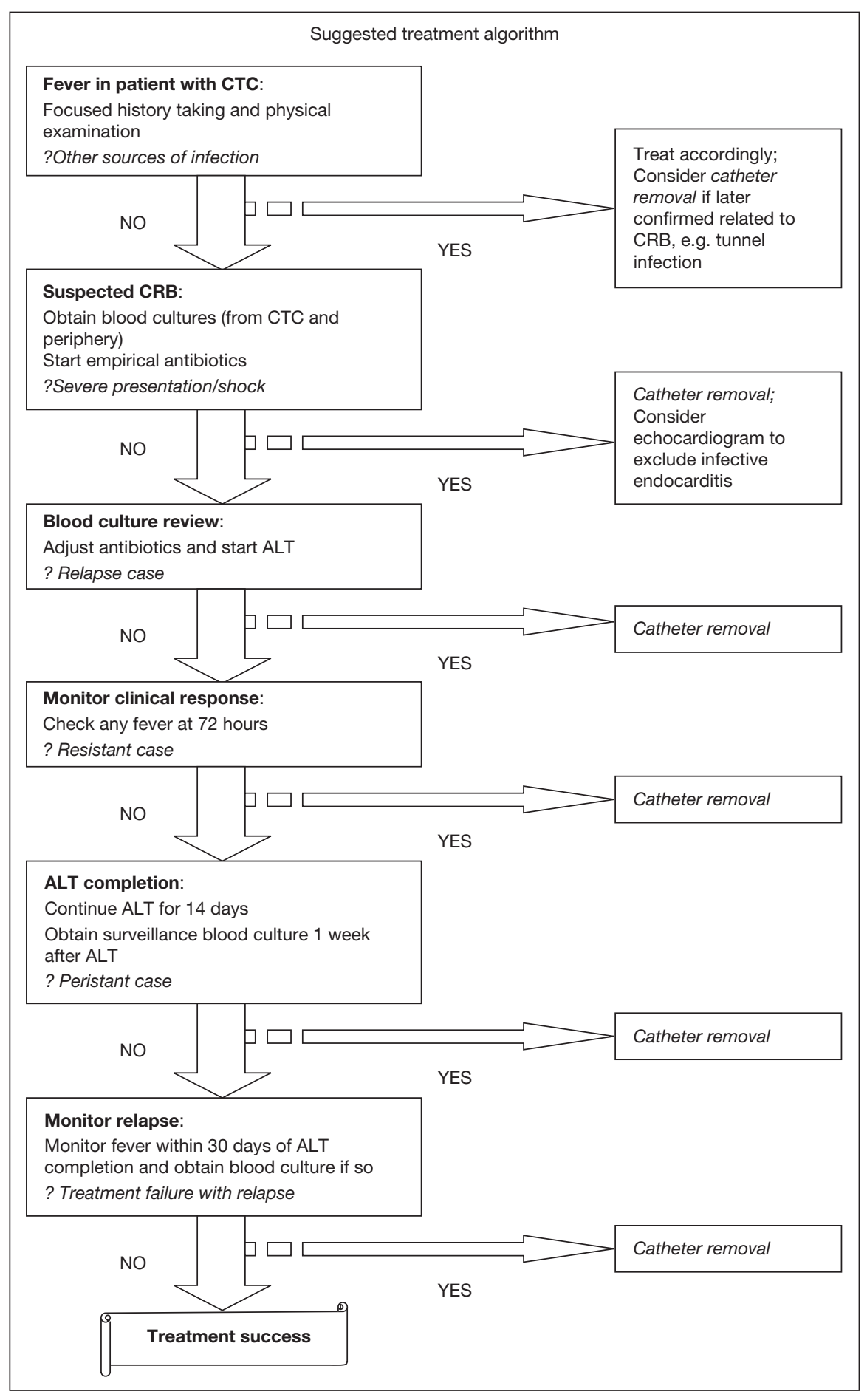

Outcome of the episodes

\begin{tabular}{llll}
\hline Treatment outcome & Study group & Control group & P value \\
\hline $\begin{array}{l}\text { success } \\
\text { failure }\end{array}$ & $30(93.8 \%)$ & $4(50 \%)$ & $<0.01$ \\
$\quad \begin{array}{l}\text { Persistent fever } \\
\quad \text { Relapse }\end{array}$ & 1 & 2 & \\
$\begin{array}{l}\text { Length of hospitalization } \\
\text { (days) }\end{array}$ & 9.4 & 2 & $<0.01$ \\
\hline
\end{tabular}

2nd Congress of the International Society

for Hemodialysis (ISHD) 
safety. Whether ALT is more cost effective than other methods like catheter exchange requires studies with head to head comparison.

16

\section{Strategic Reduction in Haemodialysis Catheter Related Bloodstream Infections}

SWY Wong, CMK Tang, YS Li, SH Lee, ILL Kong, KH Chu, HWH Chan, KL Tong

Division of Nephrology, Department of Medicine and Geriatrics, Princess Margaret Hospital, Hong Kong

Objectives: Hong Kong has high methicillin-resistant straphylococcus aureus (MRSA) prevalence since 1980s. MRSA nasal screening on hospital admissions in one public hospital revealed $10 \%$ prevalence rate in 2007. Haemodialysis patients are at high risk for catheter-related bloodstream infection (CRBSI) with a rate of 3.36 per 1000 catheter days. Mortality is estimated $12 \%-25 \%$ for each infection. This central line bundle program with MRSA decolonization therapy aimed to fight against CRBSI.

Methods: All haemodialysis patients with newly inserted jugular catheter were recruited to have MRSA nasal screening for decolonization therapy and adherence to 4 key components of the central line bundle on hand hygiene, maximum barrier precautions, chlorhexidine skin antisepsis and line necessity review. The fifth component of preferred catheter site of subclavian vein was excluded.

Results: From Feb-2008 to Jan-2009, 86 haemodialysis patients with 9615 catheter days (mean 111.8 days/patient) were analyzed. $16(18.6 \%)$ patients had history of MRSA and 23(26.7\%) patients were positive MRSA nasal carriers. They were treated successfully with decolonization protocol, and only $6(26 \%)$ patients had recurrent positive result. This resulted in a highly effective clearance rate of $74 \%$. A total of 9 CRBSI were reported. MRSA markedly decreased from 8 ( $88 \%$ in 2007$)$ to 4 ( $44 \%$ in 2008). All these except one were associated with MRSA infection history and/or history of nasal carriage explaining the major source of MRSA infection. Others without MRSA links were entrococcus faecalis, pneudomonas aureginosa and straphylococcus aureus. A reduction $(17.5 \%)$ in yearly CRBSI rate from 1.14 (2007) to 0.94 (2008) per 1000 catheter days was achieved.

Conclusions: MRSA decolonization therapy and central line bundle program can reduce the risk of CRBSI especially in MRSA infection even with extended catheter days. The investments have set against the substantial costs in treating patients with CRBSI, and ensured morbidity and mortality. Further improvements on rigorous patient education on personal hygiene and early MRSA nasal screening for all new renal patients are recommended.
17

\section{Serum Adiponectin Is Associated with Ankle-Brachial Index in Patients on Haemodialysis}

Yi-jun Zhou, Zhao-hui Ni, Jia-qi Qian

Renji Hospital, Shanghai, China

Background: Cardiovascular disease is the major cause of death in end-stage renal disease patients. Peripheral arterial disease (PAD) has not been extensively studied. Recently available data suggest that PAD is prevalent in hemodialysis (HD) patients and is a strong predictor for subsequent cardiovascular and overall mortality. Therefore, it is very meaningful to study the risk of PAD in HD patients. Adiponectin (ADPN) is a collagen-like protein synthesized by adipocytes. There are not many data on ADPN with PAD in this population.

Objectives: To clarify the risk of PAD in HD patients in our hemodialysis center and to evaluate the roles of ADPN in the development of PAD.

Methods: 116 chronic HD patients were enrolled, including 62 male patients (the average age is $56.44 \pm 14.08$ years old). ABI was used as an estimate of the presence of PAD. ADPN, high-sensitivity CRP, ferritin, hemoglobin, albumin, lipid profiles, blood glucose, blood calcium, phosphorus were measured. Logistic regression was used to estimate the association between the presence of PAD and ADPN and other potential risk factors.

Results: The incidence of PAD in our center is $18 \%$. Serum levels of ADPN were significantly lower in patients with evidence of PAD than in those without $(8.68 \pm 1.08 \mathrm{vs} 11.99 \pm 1.80 \mu \mathrm{g} / \mathrm{ml})$. Univariate analysis showed a positive correlation between $A B I$ values and serum ADPN ( $r=0.719, p=0.001)$, negative correlations between $\mathrm{ABI}$ values and hsCRP, age, blood glucose and triglyceride. Using logistic regression, an increase level of serum ADPN was associated with an odds ratio of PAD of 0.858 (95\%CI:0.852-0.975, $\mathrm{p}=0.001$ ). In addition, smoking $(\mathrm{OR}=11.117,95 \% \mathrm{CI}: 1.418-17.154, \mathrm{p}=0.022)$, blood glucose $(\mathrm{OR}=2.314,95 \% \mathrm{CI}: 1.135-4.715, \mathrm{p}=0.021)$ and triglyceride $(\mathrm{OR}=2.188,95 \% \mathrm{CI}: 1.895-5.353, \mathrm{p}=0.046)$ are found to be risk factors for PAD.

Conclusion: The incidence of PAD in our hemodialysis patients is higher than in the general population. Decreasing levels of adiponectin were associated with a significant increase in the risk of PAD. This suggests that the serum ADPN level may play a role in the atherosclerotic process of PAD. 


\section{Home-Based Therapy}

18

\section{Expanding Hemodialysis Choices: Sustaining} Home Overnight Haemodialysis

\author{
JWM Agar, RE Simmonds, JM Boddington, R Hungerford, \\ TMandic \\ Geelong Hospital, Barwon Health, Geelong, Victoria, \\ Australia
}

Some believe overnight (nocturnal) home-based hemodialysis (NHHD) to be the best dialysis option of all but, unfortunately, most still raise perceived barriers and objections to home care. Though the biochemical, physiological and financial outcomes of NHHD are now widely understood to be superior to conventional facility-based HD, doubts still abound regarding the recruitment, training, supporting and sustaining of home-based patients.

An active NHHD program has been sustained in our 'blue-collar' industrial city since 2000/2001. By 2004, we had achieved a homebased penetrance of $>25 \%$ of all HD, a rate since sustained unbroken at $25-30 \%$ (currently $27 \%$ ) of all HD and $\sim 22.5 \%$ of all dialysis including PD (currently 14\% of all dialysis). This is despite an older, more co-morbid patient group than the Australian national mean ${ }^{1}$ (see Table).

\begin{tabular}{lll}
\hline Category & $\begin{array}{l}\text { Service } \\
{[\mathrm{n}+(\%)]}\end{array}$ & $\begin{array}{l}\text { Australia } \\
{[\mathrm{n}+(\%)]}\end{array}$ \\
\hline Age $\geq 55$ & $132(81.9)$ & $7775(66.9)$ \\
Male Gender & $109(67.7)$ & $6994(60.2)$ \\
Cigarettes (current or former) & $104(64.6)$ & $6153(52.9)$ \\
Hypertension on treatment & $89(55.3)$ & $5093(50.8)$ \\
Chronic lung disease & $37(23.0)$ & $1814(15.6)$ \\
Coronary artery disease & $70(43.5)$ & $3884(33.4)$ \\
Peripheral vascular disease & $46(28.6)$ & $2179(18.8)$ \\
Diabetes (as cause or co-morbidity) & $64(39.8)$ & $4832(41.6)$ \\
\hline
\end{tabular}

Our collective, cross-service, 'home is best' attitudinal change was achieved through staff in-service discussions and active pre-dialysis patient education while the publication of outcome data has added further to the uptake stimulus. Strong, empathetic and efficient home support systems are essential, though carer-dependant NHHD is not.

The 'home is best' option (both HD and PD) is now being encouraged Australia-wide after significant NHHD program savings led the Victorian Department of Human Services to establish state funding streams and incentives for NHHD.

\footnotetext{
${ }^{1}$ http://www.anzdata.org.au
}

19

The Financial Aspect of Nocturnal Home Hemodialysis (NHHD) in Hong Kong (HK) - A Community Partnership Hemodialysis (HD) Program Reducing the Cost Of HD and Increasing Patients' Employment Income

Joseph HS Wong, Rosaline NL Yip, Agnes SC Cheung, Janet SC Li, Bonnie ML Tam, KF Chau, CS Li

Renal Unit, Department of Medicine, Queen Elizabeth Hospital, Hong Kong

Background: End stage renal disease patients with inflexible centre HD schedule are known to have poor quality of life and full time work is hardly achievable. Moreover, centre HD is expensive and staff demanding. The increasing HD demand in HK is posing stress on existing limited resources. We hypothesis that NHHD serve to meet the increasing HD demand by offering a less expensive form of HD therapy and at the same time improves patients' clinical outcome, quality of life and employment rate.

Methods and Results: Alternative night NHHD ( $8 \mathrm{hrs} / \mathrm{session})$ was developed in HK as a partnership program between HD companies, government hospital and patients. All patients have to pay for HK $\$ 4700$ per month for the rental of HD machine. 10 patients $(5 \mathrm{M}$ : $5 \mathrm{~F}$, mean age $40.5 \pm 8 \mathrm{yrs}$ ) was recruited into the NHHD program since 2007. After conversion to NHHD, all patients attained full vocational rehabilitation leading to an increased mean household income per month from $\mathrm{HK} \$ 37700 \pm 25560$ to $45122 \pm 20957$. Cost analysis study was performed among centre HD and NHHD showing NHHD was a less expensive therapy. The three years cumulative HD cost per patient for hospital HD was HK\$720000, for satellite HD was HK $\$ 549000$ and for NHHD was HK\$447000.

Conclusions: NHHD provided a less expensive HD treatment to meet the increasing HD demand with improved patients' employment income in HK.

Table 1. Cumulative Cost for HD per patient (HK\$)

\begin{tabular}{llll}
\hline & $1^{\text {st }}$ year & $2^{\text {nd }}$ year & $3^{\text {rd }}$ year \\
\hline Hospital HD (2x/wk) & 240,000 & 480,000 & 720,000 \\
Satellite HD (2x/wk) & 183,000 & 366,000 & 549,000 \\
NHHD (3.5x/wk) & 191,000 & 334,000 & 447,000 \\
\hline
\end{tabular}


20

The Response of Anaemia to Improved Kt/V in Nocturnal Home Haemodialysis with Alternate Night Schedule: Local Experience in Hong Kong

\author{
KS Fung ${ }^{1}$, HL Tang ${ }^{1}$, JHS Wong ${ }^{2}$, CMK Tang ${ }^{1}$, KH Chu',

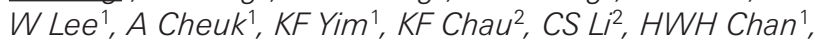 \\ KL Tong ${ }^{1}$ \\ ${ }^{1}$ Division of Nephrology, Department of Medicine and \\ Geriatrics, Princess Margaret Hospital, Hong Kong, ${ }^{2}$ Renal \\ Unit, Department of Medicine, Queen Elizabeth Hospital, \\ Hong Kong
}

Objectives: Anaemia is an established cardiovascular risk factor in patients with end-stage renal disease. Nocturnal home haemodialysis (NHHD) offers increase in uraemic solute clearance, dialysis adequacy and possibly through removal of toxins on hematopoiesis which result in better response to erythropoietin and improvement in anaemia management. We reviewed patients newly converted to NHHD in two local centres and reported on the experience.

Methods: Consecutive patients started on NHHD programme with alternate night dialysis for 6 to 8 hours ( 3.5 sessions a week) at 2 centres between 8/2006 and 4/2009 were studied retrospectively. Haemoglobin ( $\mathrm{Hb}$ ) level, erythropoietin (EPO) dosage, weekly Kt/V and iron status were determined at baseline and at 6 and 12 months.

Results: 13 patients had completed 6 months on NHHD, amongst them 10 had completed 12 months. For 13 patients at 6 months, $\mathrm{Hb}$ concentration increased from baseline $9.5 \pm 1.7$ to $11.2 \pm 1.6 \mathrm{~g} / \mathrm{dL}$, $(\mathrm{p}<0.05 \mathrm{n}=13)$, for 10 patients at 12 months, $10.2 \pm 0.8$ to $11.0 \pm 1.8$ $\mathrm{g} / \mathrm{dL},(\mathrm{p}=0.24 \mathrm{n}=10)$; despite a reduction in EPO dosage from $120.6 \pm 44.3$ to $69.2 \pm 60.9 \mathrm{U} / \mathrm{kg} /$ week, $(\mathrm{p}<0.01 \mathrm{n}=13,6$ months $)$ and $120.6 \pm 42.7$ to $70.8 \pm 80.6 \mathrm{U} / \mathrm{kg} /$ week, $(\mathrm{p}<0.05 \mathrm{n}=10,12$ months). Total 4 patients were able to be taken off EPO, two at 6 months and a further two at 12 months. Weekly $\mathrm{Kt} / \mathrm{V}$ increased from $3.61 \pm 0.98$ to $8.84 \pm 5.15(\mathrm{p}<0.01 \mathrm{n}=13,6$ months $)$ and from $3.52 \pm 1.04$ to $8.13 \pm 2.39(\mathrm{p}<0.01 \mathrm{n}=10,12$ months $)$. The iron status remained unchanged. Iron saturation at baseline was $29.2 \pm 10.8$ vs 6 months: $29.7 \pm 10.3 \%(p=0.86 \mathrm{n}=13)$; baseline vs 12 months: $29.8 \pm 11.4$ vs $28.4 \pm 12.4 \%(\mathrm{p}=0.72 \mathrm{n}=10)$ and for ferritin: baseline vs 6 months: $868.2 \pm 581.9$ vs $819.3 \pm 677.2 \mathrm{pmol} / \mathrm{L}(\mathrm{p}=0.35 \mathrm{n}=13)$ and baseline vs 12 months: $923.0 \pm 607.8$ vs $628.9 \pm 562.8 \mathrm{pmol} / \mathrm{L}(\mathrm{p}=0.093$ $\mathrm{n}=10)$.

Conclusions: Enhanced $\mathrm{Kt} / \mathrm{V}$ in NHHD is associated with improvement of anemia and reduction in requirement or even cessation of EPO usage.
21

\section{Effective Control of Serum Phosphate in Patients Receiving Nocturnal Home Hemodialysis with an Alternate Night Schedule - A Local Experience}

HL Tang ${ }^{1}$, JHS Wong ${ }^{2}$, CMK Tang ${ }^{1}, K H$ Chu $^{1}$, W Lee ${ }^{1}$, A Cheuk', KF Yim ${ }^{1}, K S$ Fung ${ }^{1}$, KF Chau ${ }^{2}, C S ~ L i{ }^{2}$, HWH Chan ${ }^{1}, K L$ Tong ${ }^{1}$

${ }^{1}$ Division of Nephrology, Department of Medicine and Geriatrics, Princess Margaret Hospital, Hong Kong, ${ }^{2}$ Renal Unit, Department of Medicine, Queen Elizabeth Hospital, Hong Kong

Objectives: Hyperphospatemia in patients with end-stage renal disease (ESRD) is associated with increase mortality. It plays an important role in the development of hyperparathyroidism and vascular calcification. This study reviews the effectiveness of nocturnal home hemodialysis (NHHD) in the control of serum phosphate in two local centres.

Methods: Thirteen patients were started on NHHD between 2006 and 2009 with an alternate night schedule (3.5 sessions/week) for 6 to 8 hours. Predialysis serum phosphate level, calcium phosphate product and dose of phosphate binders before NHHD, at 6 and 12 months, and the percentage reduction of serum phosphate during a conventional hemodialysis (CHD) session for 4-5 hours and that during a NHHD session were retrospectively reviewed.

Results: Thirteen patients have performed NHHD for 6 months and 10 of them for 12 months. For the 13 patients at 6 months, the predialysis serum phosphate level decreased significantly from baseline of $2.34 \pm 0.43$ (mean $\pm \mathrm{SD})$ to $1.56 \pm 0.39 \mathrm{mmol} / \mathrm{L}(\mathrm{p}<0.01$, $\mathrm{n}=13$ ) and for the 10 patients at 12 months, $2.31 \pm 0.44$ to $1.56 \pm 0.21$ $\mathrm{mmol} / \mathrm{L}(\mathrm{p}<0.01, \mathrm{n}=10)$. Calcium phosphate product also decreased significantly from $5.34 \pm 0.98$ at baseline to $3.68 \pm 0.80 \mathrm{mmol}^{2} / \mathrm{L}^{2}$ $(\mathrm{p}<0.01, \mathrm{n}=13)$ at 6 months, and from $5.39 \pm 1.02$ to $3.63 \pm 0.65$ $\mathrm{mmol}^{2} / \mathrm{L}^{2}(\mathrm{p}<0.01, \mathrm{n}=10)$ at 12 months. The percentage reduction of serum phosphate during a NHHD session was higher than that during a CHD session ( $60 \pm 14$ vs $48 \pm 13 \%, \mathrm{p}=0.225, \mathrm{n}=5)$ although statistically insignificant. Phosphate binder dose was reduced after NHHD and 3/13 patients (23\%) were taken off phosphate binders at 6 months and $4 / 10$ patients $(40 \%)$ at 12 months. Addition of phosphate (using Fleet enema) into the dialysate was required in $2 / 13(15 \%)$ patients due to low predialysis serum phosphate level.

Conclusions: NHHD with an alternate night schedule effectively controls serum phosphate in patients with ESRD, allowing a dose reduction and even discontinuation of phosphate binder. 


\section{Continuous Therapy and ICU}

\section{2 \\ Prolonged Catheter Survival in Patients with Acute Kidney Insufficiency on Continuous Renal Replacement Therapy Using a Less Thrombogenic Micropatterned Polymer Modification}

\section{Pascal Meier}

Centre Hospitalier du Centre du Valais, Sion, Switzerland

Continuous renal replacement therapy (CRRT) has been increasingly used in critically ill patients with acute kidney insufficiency (AKI). One of the major properties that likely influence the catheter lifespan includes its inner surface specificity. We hypothesized that the improvement of blood-surface interaction by a reactive polymer film coating might reduce thrombogenic events in the vascular access device and subsequently lead to prolonged catheter survival in the clinical setting. We therefore compared, in a randomized cross-over single-blinded study, the clinical application of two temporary catheters (TCs): 1 standard double lumen catheter (sDLC) and 1 surface modified double lumen catheter (smDLC) with identical geometry and flow design. Anticoagulation and platelet anti-aggregation were allowed. Efficacy endpoints were defined as the ability to complete 72-h CRRT without interruption due to TCs dysfunction and ability to achieve blood flow rates of ${ }^{3} 150 \mathrm{~mL} / \mathrm{min}$. Safety endpoints were defined as the occurrence of infection or bleeding. We evaluated 236 critically ill patients with AKI on CRRT (CVVHDF) with mean \pm SD age of $56.5 \pm 18.3 \mathrm{y}$. Total ultrafiltrate was $18.4 \pm 6.7$ liters, for $3.2 \pm 2.5$ liters/d. The clinical investigation revealed that the number of hours before TCs removal according to clinical requirements was significantly higher with smDLC as compared with sDLC $(116 \pm 38$ vs. $92 \pm 27$ hours; $p=0.004)$. The blood flow rate was $221 \pm 29 \mathrm{~mL} /$ $\min$ vs. $187 \pm 36 \mathrm{~mL} / \mathrm{min}$ for smDLC and sDLC; $\mathrm{p}=0.01$. TC malfunction occurred in $11 \%$ and $24 \%$ for smDLC and sDLC; $p=0.001$. Thrombosis of smDLC and sDLC was observed in 3.2 (2.7 episodes per 1,000 UCT days) vs. 7.1\% (4.2 episodes per 1,000 UCT days); $\mathrm{p}=0.001$. There was a significant difference in local infection rate as observed $(\mathrm{p}=0.002)$. Micropatterned surface coating with a polyurethane polymer significantly increased TC survival with lower dysfunction rate and better bacteriological barrier than SDLC in critically ill patients with AKI necessitating CRRT.
23

Effects of Continuous Blood Purification Therapy on Early Gut Mucosal Dysfunction in Patients with Severe Acute Pancreatitis

Jian Bin Zhang, Hua Gan, Xiao Gang Du

ChongQing Medical University, ChongQing, China

Objective: Early Gut mucosal dysfunction is one of the critical pathophysiologic disorders in patients with severe acute pancreatitis (SAP). To invest the effect of continuous blood purification(CBP) on gut mucosal dysfunction, we conducted a prospective study of 26 patients with SAP.

Method: All patients underwent CVVH for 24 hours. Permeability of the epithelial monolayer, serum concentration of diamine oxidase (DAO), D-lactate and endotoxin were used as the markers for assessment of epithelial function. Blood samples were taken from the patients at 0,6,12,24 h during CVVH therapy. Serum DAO, D-lactate and endotoxin were determined by spectrophotography. Permeability of the epithelial monolayer was assessed by transepithelial electric resistance (TEER) using 9 cultured Caco- 2 cell monolayer and the change in actin was measured by fluorometry.

Result: The Acute Physiology and Chronic Healthy Evaluation (APACHE) score was improved significantly after CVVH. Compared with normal controls, patients with SAP had increased levels of serum DAO, D-lactate, endotoxin and permeability. Peripheral filament bands become blurred, cell-cell junction loosing was also seen. After CBP treatment, serum DAO, D-lactate and permeability decreased in all patients. CBP treatment also significantly attenuated the reorganization of actin.

Conclusion: Early gut mucosal dysfunction is present in patients with SAP. CBP cannot only improve the general conditions but also effectively improves gut mucosal dysfunction. This may attenuate reorganization of actin by clearing the over-produced pro-inflammatory cytokines.

24

Individualized Bicarbonate Content in the Replacement Solution for Continuous Venovenous Hemofiltration (CVVH) with Regional Citrate Anticoagulation (RCA)

YL Cheng, KB Tai, MC Luk, KY Tsang, WY Lau, LM Hau, SM Wong, HS Chan, KK Wong, AWY Yu

Department of Medicine, Alice Ho Miu Ling Nethersole Hospital, Hong Kong

We attempted to minimize citrate-related acid-base disturbance by individualizing the bicarbonate $\left(\mathrm{HCO}_{3}\right)$ concentration in the replacement solution for CVVH treatment using RCA.

Methods: The CVVH treatment was delivered by a Gambro PRISMA machine using M60 or M100 sets with AN69 hemofilters. Blood flow was started at a rate of $120 \mathrm{~mL} / \mathrm{min}$ and increased to 150 $\mathrm{mL} / \mathrm{min}$ if tolerate.. Anticoagulant Citrate Dextrose Solution-Formula A was infused pre-filter at a fixed rate of $240 \mathrm{~mL} / \mathrm{hr}$. Three replace- 
ment solutions, namely Hemosol BO solution $\left(\mathrm{R}_{1}\right), 2.5 \%$ Dextrose in $0.45 \%$ saline $\left(R_{2}\right)$ and normal saline $\left(R_{3}\right)$, were infused post-filter. $R_{1}$ was infused via the PRISMA machine, and $R_{2}$ and $R_{3}$ were infused via the return line by separate infusion pumps. Infusion rates of $R_{1}$, $R_{2}$ and $R_{3}$ were adjusted according to the pre-treatment plasma $\mathrm{HCO}_{3}$ level and the target ultrafiltration (UF) rate (Table 1).

Premixed bag of potassium chloride in $\mathrm{R}_{2}$ was used if potassium supplement was required. Calcium chloride was infused centrally to maintain the systemic ionized calcium.

Results: 25 patients (mean age $64 \pm 14$ ) who underwent 39 CVVH sessions were observed. Six, 17, 11 and 5 CVVH treatments used the protocol A, B, C and D, respectively. Results were shown in Table 2 .

Five patients treated with protocol $\mathrm{B}$ developed plasma $\mathrm{HCO}_{3}>30$ $\mathrm{mM}$ in contrast to none in protocol $\mathrm{A}$ and $\mathrm{D}$, and 1 patient in protocol C.

Conclusions: Despite having the lowest $\mathrm{HCO}_{3}$ content in the 'mixture' of the 3 replacement solutions, a significantly higher plasma $\mathrm{HCO}_{3}$ level was observed when the pre-treatment plasma $\mathrm{HCO}_{3}$ level was $>20 \mathrm{mM}$ and the UF rate was set at $1500 \mathrm{~mL} / \mathrm{hr}$. Our findings suggest the $\mathrm{HCO}_{3}$ content in the replacement solution needs to be individualized to the pre-treatment plasma $\mathrm{HCO}_{3}$ level to minimize metabolic alkalosis in CVVH treatment using RCA.

Table 1

\begin{tabular}{|c|c|c|c|c|c|c|}
\hline \multirow[t]{2}{*}{ Protocol } & \multirow{2}{*}{$\begin{array}{l}\text { Pre-treatment } \\
\text { plasma } \\
\mathrm{HCO}_{3}, \mathrm{mM}\end{array}$} & \multirow{2}{*}{$\begin{array}{l}\text { Target } \\
\mathrm{UF}, \\
\mathrm{mL} / \mathrm{hr}\end{array}$} & \multicolumn{3}{|c|}{ Infusion rate, $\mathrm{mL} / \mathrm{hr}$} & \multirow{2}{*}{$\begin{array}{l}\mathrm{HCO}_{3} \\
\text { level in the } \\
\text { mixture of } \\
\mathrm{R}_{1}, \mathrm{R}_{2} \text { and } \\
\mathrm{R}_{3}, \mathrm{mM}\end{array}$} \\
\hline & & & $\mathrm{R}_{1}$ & $\mathrm{R}_{2}$ & $\mathrm{R}_{3}$ & \\
\hline A & $\leq 20$ & 1500 & 850 & 100 & 250 & 22.7 \\
\hline B & $>20$ & 1500 & 650 & 100 & 450 & 17.3 \\
\hline $\mathrm{C}$ & $\leq 20$ & 2000 & 1200 & 150 & 350 & 22.6 \\
\hline $\mathrm{D}$ & $>20$ & 2000 & 1000 & 150 & 500 & 19.4 \\
\hline
\end{tabular}

25

\section{Long-Term Outcome of Survivors of CRRT in Children}

\author{
WKY Chan, KW Lee, WF Hui, CW Law, TY Miu, WH Lee \\ Department of Paediatrics, Queen Elizabeth Hospital, \\ Hong Kong
}

Introduction: With the advancement in medical technology, various modalities of CRRT become feasible in children. However, their impact in modifying the long-term outcome of survivors is not well studied.

Methods: We retrospectively reviewed our patients who had CRRT between January 1998 and December 2008. Their outcomes were reviewed at 6 months, one year after CRRT and at last follow up. Late survivors are defined as survival beyond one year after CRRT.

Results: In the study period, 37 patients were recruited. Twentyone patients $(57 \%)$ survived the acute event. Among these early survivors, fourteen patients were male and seven were female. The mean age of patients in the paediatric group was $7.7 \pm 6$ years and the median age at CRRT for neonate was day 2 of life (range: 2-15 days). One patient was followed less than 6 months and was excluded from further review. At 6 month after CRRT, 4 patients died of their underlying diseases, 4 patients were still receiving dialysis therapy and survived the acute event. Twelve patients had normalized their renal functions. At the one-year assessment, three more patients died, making a total of 7 deaths $(33.33 \%)$ among the early survivors in the first year after CRRT. Thirteen patients (62\%) survived beyond one year. The mean duration of follow up of these late survivors was $5.5 \pm 2.6$ years. At final assessment, three patients (14.3\%) were in Chronic Kidney Disease (CKD) stage V and were on renal replacement therapy, 2 patients had CKD stage II and one was in CKD stage III.

Conclusion: With the support of CRRT, patients survived the acute life-threatening event but $1 / 3$ of them still succumbed within the first year and was related to their underlying diseases. Monitoring of renal function in the survivors was recommended as it may continue to deteriorate years later.

Table 2

\begin{tabular}{|c|c|c|c|c|c|c|}
\hline Protocol & Filter life, hr & \multicolumn{4}{|c|}{ Plasma $\mathrm{HCO}_{3}$ levels } & End \\
\hline A & $32.3 \pm 11.5$ & $18.6 \pm 3.7$ & $17.9 \pm 3.4^{\mathrm{a}}$ & $21.5 \pm 4.5^{\mathrm{d}}$ & $21.5 \pm 2.9^{\mathrm{g}}$ & $26.6 \pm 2.0$ \\
\hline $\mathrm{C}$ & $26.7 \pm 12.6$ & $18.1 \pm 3.3$ & $19.4 \pm 1.9^{\mathrm{c}}$ & $21.9 \pm 2.5^{\mathrm{f}}$ & $23.0 \pm 2.6^{\mathrm{i}}$ & $23.4 \pm 3.5^{\mathrm{k}}$ \\
\hline D & $20.2 \pm 4.8$ & $22.8 \pm 2.0$ & $21.6 \pm 3.7$ & $21.7 \pm 5.1$ & $23.6 \pm 3.3$ & $21.7 \pm 5.0^{\mathrm{m}}$ \\
\hline
\end{tabular}

a vs b, b vs c, j vs k, j vs m: $p<0.001$; d vs e, e vs f: $p<0.05$; g vs h, h vs i: $p<0.01$ 


\section{6}

Review on the Use of Continuous VenoVenous Haemofiltration (CVVH) in PICU Supported by a Tertiary Renal Referral Centre in Hong Kong

\author{
NC Fong, CY Chow, ALTMa, PY Loung, KC Tse, WM Lai, \\ YW Hui, MC Chiu \\ Princess Margaret Hospital, Hong Kong
}

Aim: To review the use of CVVH in PICU supported by a tertiary Paediatric renal centre.

Method: Patients with CVVH done between 2000 to 2009 were reviewed. Demographic data, indications for CVVH, clinical condition and co-morbidities, CVVH setting, CVVH dosage, complications and outcome were studied.

Results: 24 patients were reviewed. Mean age was $6.3+/-7.4 \mathrm{yr}$ (0.01-23.49) with M:F 16:8. Mean body weight was $23.5+/-21.5 \mathrm{~kg}$ (3.12-72). Mean urea and creatinine concentration before CVVH were $18.9+/-10.6 \mathrm{mmol} / \mathrm{L}(2.5-42)$ and $283+/-251.9 \mathrm{umol} / \mathrm{L}(87-1183)$. Mean GFR was $25.6+/-15.8 \mathrm{ml} / \mathrm{min} / 1.73 \mathrm{~m}^{2}$ (2-57.8). Indications for CVVH were: Haemolytic Uraemic Syndrome (HUS) (7), tumor lysis syndrome (1), Wegener's granulomatosis (1), ARF post renal transplant (1), ARF with dysplastic kidney (1), rhabdomyolysis (1), multiple organ failure (7) and inborn error of metabolism (IEM) (5). Vascular assess was obtained percutaneously with 4 subclavian and 20 femoral approach. Blood flow rate was $20-150 \mathrm{ml} / \mathrm{min}$ with replacement fluid rate $150-1500 \mathrm{ml}$ per hour. Mean CVVH dose was $26.5+/-10.5 \mathrm{ml} / \mathrm{kg} / \mathrm{hr}$ (10.1-50.4). Mean duration of CVVH was 148.3 +/- $130.1 \mathrm{hr}$ (4-459) with mean CVVH filter life span $48.8 \mathrm{hrs}$. 3 patients had mild blood oozing and 7 patients had transient hypotension on commencement of CVVH. 13 out of 24 patients $(54.2 \%)$ survived with $69 \%(9 / 13)$ of them below 2 years old. These included all HUS patients, tumor lysis syndrome, 2 IEM, rhabdomyolysis, ARF post renal transplant and ARF with dysplastic kidney. The youngest patient aged 5 days of $3.12 \mathrm{~kg}$ responded well to CVVH. Good survival for CVVH patients was associated with isolated renal problem and less inotropic support . $(\mathrm{p}<0.05)$

Conclusion: CVVH is an effective means for renal support with underlying renal and metabolic problems in Paediatric patients. Complications are few in established PICU with good renal support. Early CVVH may improve survival of those with multiple organ failure.

\section{Nursing Matters}

\section{7 \\ Pre-End Stage Renal Disease Education Increases Permanent Access Use at Initiation of Dialysis}

\author{
Lydia Lim, Jason Choo, Koh Ming Yong, Hui Lin Choong \\ Singapore General Hospital, Singapore
}

Introduction: Pre-ESRD education plays an important role in assisting the patient with Chronic Kidney Disease (CKD) and their family in making an informed choice on renal replacement therapy (RRT). It provides objective information on treatment alternatives and assists them in choosing a treatment suited to their medical condition and lifestyle.

Methodology: Pre- ESRD education is conducted by renal coordinators (RCs) who are registered nurses with renal experience. All patients progressing to end stage renal disease will be asked to attend a structured educational programme. Upon receiving referral from nephrologists, the RC would initiate individualized sessions either at outpatient or inpatient level. The topics covered includes the disease process, the different treatment modalities including renal transplantation, required surgical procedures for dialysis and application to subsidy programmes.

Results: All 347 chronic kidney disease patient aged $60+/-11.6$ years (range 23-89) initiated on dialysis from Jan 2008 to Dec 2008 were included in this survey. $53 \%$ were males. Ethnic distribution was as follows: Chinese $68 \%$, Malay $24 \%$, Indian $7 \%$ and $1 \%$ others. The main cause of ESRD was diabetes nephropathy (65\%).

Demographics \& Clinical Characteristics

\begin{tabular}{ll}
\hline & $\begin{array}{l}\text { Overall } \\
\mathrm{n}=347\end{array}$ \\
\hline Age, Mean + SD (years) & $60 \pm 11.6$ \\
Male (\%) & 53 \\
Female & 47 \\
Race (\%) & \\
- Chinese & 68 \\
- Malay & 24 \\
- Indian & 7 \\
- Others & 1 \\
Cause of ESRD (\%) & \\
- Diabetic Nephropathy & 64.8 \\
- Hypertension & 9.8 \\
- Glomerulonephritis & 14.1 \\
- Obstruction & 2 \\
- Polycystic Kidney Disease & 1.7 \\
- Others & 7.6 \\
First RRT modality at onset of ESRD (\%) & \\
- Haemodialysis & 94.2 \\
- Peritoneal Dialysis & 5.8 \\
Access used for 1st dialysis (\%) & \\
- Arteriovenous Fistula or graft & 7.5 \\
- Tenckhoff catheter & 5.8 \\
- Catheter & 86.7
\end{tabular}


Comparison between patients with pre-ESRD education $<3$ months or no education and pre-ESRD education $\geq 3$ months prior to initiation of Haemodialysis

\begin{tabular}{llll}
\hline & $\begin{array}{l}\text { Group 1 } \\
\mathrm{n}=240\end{array}$ & $\begin{array}{l}\text { Group 2 } \\
\mathrm{n}=87\end{array}$ & p-value \\
\hline Age, Mean (years) & 60 & 61 & $\mathrm{NS}$ \\
Male Gender (\%) & 52 & 57.5 & $\mathrm{NS}$ \\
Race (\%) & & & \\
- Chinese & 67.9 & 65.5 & $\mathrm{NS}$ \\
- Malay & 24.6 & 21.8 & \\
- Indian & 6.7 & 9.2 & \\
- Others & 0.8 & 3.5 & \\
Cause of ESRD (\%) & & & \\
- Diabetic Nephropathy & 64.6 & 64.4 & \\
- Hypertension & 7.1 & 17.2 & \\
- Glomerulonephritis & 15.4 & 11.5 & \\
- Obstruction & 2.5 & 1.1 & \\
- Polycystic Kidney Disease & 1.7 & 2.3 & \\
- Others & 8.7 & 3.5 & \\
Permanent Vascular Access used for & & & \\
1st dialysis (\%) & & & \\
- Arteriovenous Fistula or graft & 3.8 & 19.5 & NS \\
Mortality at 90 days after initiating & & & \\
haemodialysis (\%) & 7.9 & 4.6 & NS \\
Long Term Plan (\%) & & & \\
- Haemodialysis & 93.8 & 90.8 & \\
- Peritoneal Dialysis & 6.2 & 9.2 & \\
\hline
\end{tabular}

Most ( 327;94\%) were initiated on haemodialysis. Majority of patients $(240 ; 73 \%)$ had education less than 3 month or no education before initiation of dialysis (Gp1). The remainder $(27 \%)$ were referred electively and educated at least 3 months prior to initiation (Gp 2). Only 3.8\% of patients in Gp 1 had permanent vascular access created prior to initiation of dialysis as compared with $19.5 \%$ in $\mathrm{Gp} 2$. Patients with Pre-ESRD education more than three months before initiation of dialysis are more likely $(p<0.000$, odds ratio 5.14) to have early permanent vascular access created.

Conclusion: Patients should be referred early for pre-ESRD education to have a better planned start leading to better therapy outcome.

28

\section{An Unique Nursing Experience in Establishing Nocturnal Home Hemodialysis (NHHD) Program in Hong Kong (HK)}

Agnes SC Cheung, Janet SC Li, Rosaline NL Yip, Bonnie ML Tam, Joseph HS Wong, KF Chau, CS Li

Renal Unit, Queen Elizabeth Hospital, Hong Kong

Background: Peritoneal dialysis (PD) first policy adopted in HK resulted in a very high $\mathrm{PD}$ proportion of $80 \%$ in the dialysis population. With increasing number of PD failure, there is an accompanying increasing demand on HD. NHHD therapy has demonstrated superior clinical benefits at overseas centers and is a potential home dialysis modality to solve the HD demand in HK. However, there are cultural, economical and social barriers which the nurses need to overcome to establish the NHHD program in HK.
Methods and Results: The pioneer program has been launched in Queen Elizabeth Hospital since May 2007. 10 patients (5M: 5F) with age of 22-53 (mean: 40.5) were recruited for NHHD. With pre training assessment, patients and partners were assessed for physical, psychological, social and cognitive competency for this self-help program. Home visit with advice on modification and renovation of home environment is the essence of success in the crowded living environment in HK. The mean household area of NHHD patients was $635.7+233.3$ sq feet. Flexible training schedule was offered to facilitate effective training to NHHD patients with busy working schedule. All patients resumed full time work after conversion to NHHD.

Conclusion: Nocturnal home hemodialysis is a feasible and suitable program to meet the increasing HD demand in HK.

29

\section{Comparison of Recirculation and Dialysis Adequacy between Antegrade and Retrograde Cannulations for Hemodialysis \\ Sung Kyung Kim², Hyun Kyung Kim', Ja Young Lee1, Young Soo Kim', Sun Ae Yoon'1, Young OK Kim¹, Byung Kee Bang ${ }^{3}$ \\ ${ }^{1}$ The Catholic University of Korea, Seoul, Republic of Korea, ${ }^{2}$ Uijeongbu St. Mary's Hospital, Uijeongbu, Republic of Korea, ${ }^{3}$ Hanbit Sungse Hospital, Pyungtaek, Republic of Korea}

Purpose: As a cannulation method in hemodialysis (HD) patients having arteriovenous fistula (AVF), retrograde direction (opposite direction between arterial and venous cannulations) is preferred and antegrade direction (same direction) is not commonly used because of probability of recirculation and subsequent decreased dialysis adequacy. Antegrade cannulation has longer segment for cannulation and was more comfortable and less painful, compared to retrograde cannulation. This study was performed to compare recirculation and dialysis adequacy between antegrade and retrograde cannulations in hemodialysis patients.

Methods: This study enrolled 36 chronic renal failure patients who received HD 3 times weekly for more than 3 months through AVF. We performed hemodialysis with retrograde cannulation for 4 weeks and then switched it to antegrade cannulation. We compared recirculation ratio and dialysis adequacy between the two cannulation methods. AVF blood flow and recirculation ratio were measured by ultrasonic dilution method and $\mathrm{Kt} / \mathrm{V}$ was measured by using single pool urea kinetics model.

Results: The length between arterial and venous needle tips was shorter in antegrade cannulation than retrograde cannulation $(7.8 \pm 3.5$ cm vs. $10.2 \pm 3.3 \mathrm{~cm}, \mathrm{p}<.001)$. Recirculation did not occur in both cannulation methods. There was no difference in $\mathrm{Kt} / \mathrm{V}$ between both cannulation methods $(1.43 \pm 0.14$ vs. $1.46 \pm 0.19, p=.067)$.

Conclusion: Although the length between arterial and venous needle tips was shorter in antegrade cannulation than retrograde cannulation, both cannulation methods did not make recirculation in HD patients with well-functioned AVF. And there was no difference in dialysis adequacy between the two methods. 
Miscellaneous

\section{0}

Severe 25 (OH) D Vitamin Deficiency - Continuous Problem from Dialysis to Transplantation

\section{Barbara Grandtnerova, Ludovit Laca}

Transplant Centrum, Faculty Hospital Martin, Martin, Slovakia

Background: Serum 25(OH)D levels reflect vitamin D body stores. Growing body of evidence links 25D deficiency with mortality rates in general as well as in chronic kidney disease population.

Aim: to evaluate $25(\mathrm{OH}) \mathrm{D}$ status in kidney transplant recipients immediately after transplantation as well as after medium time follow up.
Patients and Methods: 120 patients, 50 immediately after transplantation, 70 more at least 3 year after transplantation were evaluated. Serum $25(\mathrm{OH}) \mathrm{D}$ levels were measured using chemiluminescence assay.

Results: Immediately after transplantation, mean \pm SD values of $25(\mathrm{OH}) \mathrm{D}$ were $14,2 \pm \mathrm{ng} / \mathrm{ml}$. Severe deficiency $(<5 \mathrm{ng} / \mathrm{ml})$ was present in $11 \%$, deficiency $(5-15 \mathrm{ng} / \mathrm{ml})$ in $56 \%$ and insufficiency (16$30 \mathrm{ng} / \mathrm{ml}$ ) in another $33 \%$ of patients. Kidney transplantation itself did not correct $25(\mathrm{OH}) \mathrm{D}$ status, mean serum levels felt after at least 3 years of follow up without supplementation, however not significantly, to $11,3 \pm 6,9 \mathrm{ng} / \mathrm{ml}, \mathrm{p}=0,16$. Supplementation with 500 $1000 \mathrm{IU}$ of cholecalciferol daily (doses recommended in treatment of osteoporosis) improved 25(OH)D significantly (to $18,3 \pm 7 \mathrm{ng} / \mathrm{ml}$ ), $\mathrm{p}<0.001)$, however, normal levels of $25(\mathrm{OH}) \mathrm{D}$ reached $3 \%$ of patients only.

Conclusion: Vitamin 25(OH)D deficiency transmits from dialysis period to period after kidney transplantation. Spontaneous correction is not likely; doses of cholecalciferol recommended for osteoporosis prevention/therapy are not sufficient enough. In the view of recent knowledge about cardiovascular effects of vitamin D, this fact necessitates our attention. 


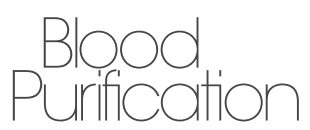

Agar, J.W.M. 11, 18

Bang, B.K. 29

Becker, C.L. 5

Boddington, J.M. 18

Cass, A.M. 11

Chan, C.K. 15

Chan, H.S. 24

Chan, H.W.H. 16, 20, 21

Chan, S.K. 15

Chan, W.K.Y. 25

Chau, K.F. 19, 20, 21, 28

Chazot, C. 12

Che, X.J. 7

Cheng, Y.L. 24

Cheuk, A. 20, 21

Cheung, A.S.C. 19, 28

Chiu, M.C. 26

Choo, J. 27

Choong, H.L. 9, 27

Chow, C.C. 15

Chow, C.Y. 26

Chow, K.-M. 2

Chu, K.H. 16, 20, 21

Dakshina Murty, K. 13

Du Toit, D. 5

Du, X.G. 14, 23

Eris, J. 11

Fong, N.C. 26

Fung, K.S. 20, 21

Gallagher, M.P. 11

Gan, H. 14, 23

Grandtnerova, B. 30

Gu, L.Y. 7
Numbers refer to abstract numbers
Hau, L.M. 24

Hawley, C. 11

Howard, K. 11

Hui, W.F. 25

Hui, Y.W. 26

Hungerford, R. 18

Hurot, J.M. 12

Jardine, M.J. 11

Jean, G. 12

Kairaitis, L. 8

Katz, I. 5

Kaul, A. 10

Kerr, P. 11

Kim, H.J. 3

Kim, H.K. 29

Kim, S.G. 3

Kim, S.J. 3

Kim, S.K. 29

Kim, Y.O. 29

Kim, Y.S. 3, 29

Kong, I.L.L. 16

Kwan, B. 2

Laca, L. 30

Lai, W.M. 26

Lau, W.Y. 24

Law, C.W. 25

Lee, J.Y. 29

Lee, K.W. 25

Lee, S.H. 16

Lee, W. 20, 21

Lee, W.H. 25

Leung, C.-B. 2

Li, C.S. 19, 20, 21, 28

Li, J.S.C. 19,28

Li, P. 2

Li, Y.S. 16

Lim, C.S. 3
Lim, L. 27

Lo, H.K.S. 15

Lorriaux, C. 12

Loung, P.Y. 26

Luk, M.C. 24

Ma, A.L.T. 26

Mandic, T. 18

Mayor, B. 12

Meier, P. 1, 22

Miu, T.Y. 25

Mo, K.L.S. 15

Nealon, A. 4

Nguyen, H. 4

Ni, Z. 6, 7, 17

Park, J.S. 3

Perkovic, V. 11

Qian, J. 6, 7, 17

Ram Rapur, V. 13

Reddy Chinthaparthi, P. 13

Reddy Pamidi, N. 13

Sharma, R.K. 10

Shin, Y. 3

Simmonds, R.E. 18

Singh, A. 10

Song, Y.R. 3

Srivastava, A. 10

Stack, A.G. 4

Szeto, C.C. 2

Tai, K.B. 24

Tam, B.M.L. 19, 28

Tan, C.H.R. 9

Tang, C.M.K. 16, 20, 21

Tang, H.L. 20, 21
Terrat, J.C. 12

Tong, K.L. 16, 20, 21

Tripathi, R. 10

Tsang, K.Y. 24

Tse, K.C. 26

Vanel, T. 12

Vo-Van, C. 12

Wall, C.A.M. 4

Wong, C.K. 15

Wong, J.H.S. 19, 20, 21, 28

Wong, K.K. 24

Wong, K.S. 15

Wong, S.M. 24

Wong, S.W.Y. 16

Yan, Y.C. 6, 7

Yim, K.F. 20, 21

Yip, R.N.L. 19, 28

Yong, K. 8

Yong, K.M. 27

Yong, O.L. 9

Yoon, S.A. 29

Yu, A.W.Y. 24

Yuill, E. 8

Zhang, J.B. 14, 23

Zhou, Y. 17

Zhu, M. 6

\section{KARGER}

(C) 2009 S. Karger AG, Basel

Fax +4161306 1234

E-Mail karger@karger.ch

www.karger.com 